Kun Dai

Yunbo Li

http://dx.doi.org/10.21278/brod72206

ISSN 0007-215X

eISSN $1845-5859$

\title{
EXPERIMENTAL AND NUMERICAL INVESTIGATION ON MANEUVERING PERFORMANCE OF SMALL WATERPLANE AREA TWIN HULL
}

UDC 629.5.017.3:629.5.022.25

Original scientific paper

\begin{abstract}
Summary
Free running model tests and a system-based method are employed to evaluate maneuvering performance for a Small Waterplane Area Twin Hull (SWATH) ship in this paper. A 3 degrees of freedom Maneuvering Modeling Group (MMG) model is implemented to numerically simulate the maneuvering motions in calm water. Virtual captive model tests are performed by using a Reynolds-averaged Navier-Stokes (RANS) method to acquire hydrodynamic derivatives, after a convergence study to check the numerical accuracy. The turning and zigzag maneuvers are simulated by solving the maneuvering motion model and the predicted results agree well with the experimental data. Moreover, free running model tests are carried out for three lateral separations and the influence of the lateral separations on maneuvering performance is investigated. The research results of this paper will be helpful for the maneuvering prediction of the small waterplane area twin hull ship.
\end{abstract}

Key words: SWATH; Maneuvering performance; MMG model; RANS

\section{Introduction}

The Small Waterplane Aera Twin Hull (SWATH) is a type of widely used innovative displacement ship. Usually, a SWATH ship consists of two fully submerged bodies and slender struts which extend upwards above the waterline and connect the underwater parts with the superstructure [1]. Compared with conventional ships, the SWATH shows better performance in ride quality, human habitability and seakeeping behavior benefiting from its special configuration. In the past decades, numerous studies have been done on hydrodynamic performance of the SWATH [2-5]. However, most studies focus on the calm water resistance and ship motions under wave conditions, while researches on maneuverability are rather scare in literature. Since maneuverability is crucial to navigation safety for a ship, it is essential to investigate the maneuverability of the SWATH.

In the field of maneuverability research, free running model tests are always regarded as the most dependable way to estimate ship maneuverability, because they use a direct method 
which is close to the reality. Besides, the most popular numerical method for prediction of ship maneuverability is the system-based method, which simulates maneuvering motions with a mathematical model, like the Abkowitz model [6] or the Maneuvering Modeling Group (MMG) model [7]. The hydrodynamic coefficients in mathematical model can be easily obtained from empirical formulas [8-10]. However, most of the empirical formulas are generated on the basis of monohulls databases. These empirical formulas are effective to apply for the conventional ships, but may not useful so much for the unconventional SWATH ship due to the distinct difference in ship shape. In the last few decades, more and more researchers prefer using CFD simulations of captive model tests for determining the hydrodynamic coefficients. He et al. (2016) [11] performed standard free running maneuvers of the KVLCC2 ship model using MMG mathematical model with linear hydrodynamic derivatives generated by Planar Motion Mechanism (PMM) simulations. Liu et al. (2018) [12] conducted virtual captive of KCS ship using unsteady RANS approach to get complete hydrodynamic derivatives in the third order Abkowitz model and predicted the turning and zigzag maneuvers. Sukas et al. (2019) [13] investigated maneuvering performance of a twinpropeller twin-rudder surface combatant by utilising unsteady RANS approach. Static drift, planar motion mechanism tests, self-propulsion and static rudder tests have been simulated to generate maneuvering coefficients. Sakamoto et al. (2019) [14] conducted CFD simulations for KVLCC2 tanker hull to get full sets of necessary maneuvring parameters in the 3-DOF MMG mathematical model. Ardeshiri et al. (2020) [15] numerically simulated PMM tests for a prolate spheroid under water vehicle using CFD method and discussed the effect of the flow velocity, frequency and amplitude on maneuvering coefficients. Overall, CFD-based virtual captive model tests have been successfully applied to determine hydrodynamic derivatives as well as interaction coefficients among hull, propeller and rudder.

The present study aims to numerically predict the maneuvering performance of a SWATH ship in a practical and convenient way at the initial design stage. A system-based method with MMG mathematical model is adopted for free running simulation. Because estimation of hydrodynamic derivatives as well as hull-propeller-rudder interaction coefficients with CFD technique requires extensive simulations of model tests, which is timeconsuming. Therefore, in this study, the hydrodynamic derivatives are obtained through CFD simulations of oblique towing tests and circular motion tests for SWATH hull without rudders and propellers, while hydrodynamic forces due to propeller and rudder are computed by empirical methods in order to reduce the computational cost. Free running model test are carried out for three lateral separation layouts of the SWATH. Numerical simulations of maneuvering motions are compared to the experimental results. The maneuverability characteristics of the SWATH with varying transverse locations of demihulls are discussed according to the experimental data.

\section{Mathematical model}

\subsection{Coordinate systems and motion equations}

The maneuvering motion in calm water is described in two right-handed coordinate systems as shown in Fig. 1. The ship motion is defined in the earth-fixed coordinate system $O_{0}-X_{0} Y_{0} Z_{0}$, where $O_{0}-X_{0} Y_{0}$ plane is located on the still water surface with positive $Z_{0}$ axis pointing downwards. The hydrodynamic forces are defined in the moving ship-fixed coordinate system $G-x y z$ with positive $z$ axis pointing downwards. Its origin $G$ is taken at the center of gravity of ship. The $x$ axis and $y$ axis point towards the bow and starboard, respectively. $\delta_{(P)}$ and $\delta_{(S)}$ represent the angle of port and starboard rudder, respectively. $\psi$ 
is the heading angle. The ship sailing speed $U$ is defined by $U=\sqrt{u^{2}+v^{2}}$, where $u$ and $v$ stand for velocity components of $U$ along the $x$ and $y$ axis, respectively. In addition, $r$ is the yaw rate and $\beta=\tan ^{-1}(-v / u)$ is the drift angle.

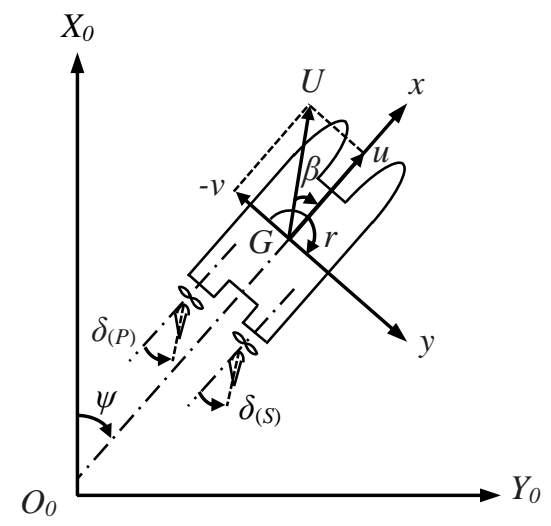

Fig. 1 Coordinate systems

Following the concept proposed by the Maneuvering Modeling Group (MMG) of Japan [16], the equations of 3-DOF maneuvering motion are expressed as:

$$
\left.\begin{array}{l}
\left(m+m_{x}\right) \dot{u}-\left(m+m_{y}\right) v r=X_{P}+X_{R}+X_{H} \\
\left(m+m_{y}\right) \dot{v}+\left(m+m_{x}\right) u r=Y_{P}+Y_{R}+Y_{H} \\
\left(I_{z}+J_{z}\right) \dot{r}=N_{P}+N_{R}+N_{H}
\end{array}\right\}
$$

In Eq. (1), $X$ and $Y$ are the hydrodynamic force components in the $x$ and $y$ directions, while $N$ denotes the yaw moment about the $z$ axis. Subscripts $P, R$ and $H$ indicate propeller, rudder and hull, respectively. $m$ is the mass of ship while $m_{x}$ and $m_{y}$ are the added mass. $I_{z}$ and $J_{z}$ are the moment and added moment of inertia about the $z$ axis.

\subsection{Hull forces and moment}

According to Yasukawa et al. (2015) [7], the hull forces and moment ( $X_{H}, Y_{H}$ and $N_{H}$ ) are described as:

$$
\left.\begin{array}{l}
X_{H}=-R_{0}+X_{v v} v^{2}+X_{v r} v r+X_{r r} r^{2}+X_{v v v v} v^{4} \\
Y_{H}=Y_{r} r+Y_{v} v+Y_{v v v} v^{3}+Y_{v r r} v r^{2}+Y_{v v r} v^{2} r+Y_{r r r} r^{3} \\
N_{H}=N_{r} r+N_{v} v+N_{v v v} v^{3}+N_{v r r} v r^{2}+N_{v v r} v^{2} r+N_{r r r} r^{3}
\end{array}\right\}
$$

where $R_{0}$ represents hull resistance in straight moving. On the right side of the Eq. (2), symbols with subscripts $u, v$ and $r\left(X_{v v}, X_{v r}, Y_{v}, Y_{v r r}, N_{v}, N_{v v r}\right.$, etc.) are called the hydrodynamic derivatives for maneuvering motion. In this study, these hydrodynamic derivatives are derived from virtual captive model test using CFD code, which is presented in Section 5.1. 


\subsection{Propeller forces and moment}

For a SWATH ship of twin-propeller twin-rudder (TPTR) configuration, propeller forces and moment $\left(X_{P}, Y_{P}\right.$ and $\left.N_{P}\right)$ are expressed as:

$$
\left.\begin{array}{l}
X_{P}=\rho\left[\left(1-t_{P(S)}\right) n_{(S)}^{2} D_{P(S)}^{4} K_{T(S)}+\left(1-t_{P(P)}\right) n_{(P)}^{2} D_{P(P)}^{4} K_{T(P)}\right] \\
Y_{P}=0 \\
N_{P}=\rho\left[y_{P(S)}\left(1-t_{P(S)}\right) n_{(S)}^{2} D_{P(S)}^{4} K_{T(S)}+y_{P(P)}\left(1-t_{P(P)}\right) n_{(P)}^{2} D_{P(P)}^{4} K_{T(P)}\right]
\end{array}\right\}
$$

In Eq. (3), superscripts $(S)$ and $(P)$ refer to the starboard and port sides, respectively. $t_{P}$ means the thrust deduction, and it is assumed to keep constant in straight moving and maneuvering motion for simplicity. Kulczyk et al. (2014) [17] showed that for a TPTR ship $t_{P} \approx 0.2$ and similar result was reported by Sukas et al. (2019) [13]. Thus, $t_{P(P)}=t_{P(S)}=0.2$ is adopted in this study. In addition, $y_{P}$ is the location of propeller in lateral direction and $y_{P(P)}=-y_{P(S)}$ because the two propellers are symmetric to the centerline of the ship. $n$ and $D_{P}$ are the propeller revolution and diameter, respectively. The two propellers are identical in geometry $\left(D_{P(P)}=D_{P(S)}\right)$ and they rotate at the same revolution rate $\left(n_{P(P)}=n_{P(S)}\right)$ but in opposite direction, therefore the integral propeller lateral force can be negligible, i.e. $Y_{P}=0$.

Commonly, the propeller thrust coefficient $K_{T}$ is approximately expressed by 2 nd polynomials of the advance ratio $\left(J_{P}\right)$ as follows:

$$
K_{T(S),(P)}=k_{2}\left(J_{P(S),(P)}\right)^{2}+k_{1} J_{P(S),(P)}+k_{0}
$$

where $k_{2}, k_{1}$ and $k_{0}$ are coefficients representing $K_{T}$. Referring to Khanfir et al. (2011) [18, 19], $J_{P}$ is expressed for a TPTR ship as:

$$
J_{P(S),(P)}=\frac{\left(u+y_{P(S),(P)} r\right)\left(1-w_{P(P),(S)}\right)}{n D_{P}}
$$

Here, $\left(u+y_{P(S),(P)} r\right)$ represents the variation due to yaw rate and propeller lateral location. Moreover, $w_{P}$ is the wake coefficient in maneuvering. It is assumed that the wake coefficient on port and starboard sides are the same for simplicity, i.e. $w_{P}=w_{P(P)}=w_{P(S)}$. Generally, the $w_{P}$ changes in maneuvering motions, and it can be evaluated based on the wake coefficient in straight advancing $\left(w_{P 0}\right)$ as follows [20]:

$$
\begin{aligned}
& w_{P} / w_{P 0}=\exp \left(-4 \beta_{P}^{2}\right) \\
& \beta_{P}=\beta-\frac{x_{P}}{L} r^{\prime}
\end{aligned}
$$

where $\beta_{P}$ denotes the geometrical inflow angle to propeller, $x_{P}$ is the longitudinal coordinate of propeller. It should be noted that $\beta_{P}=\beta_{P(P)}=\beta_{P(S)}$ because 
$x_{P}=x_{P(P)}=x_{P(S)}$ according to Eq. (7). In this study, $w_{P 0}$ is estimated using the regression formula based on a database of ships, which is expressed as follows according to Kijima et al. (1990) [9]:

$$
w_{P 0}=0.5 C_{b}-0.05
$$

\subsection{Rudder forces and moment}

For a TPTR ship, the rudder forces and moment $\left(X_{R}, Y_{R}\right.$ and $\left.N_{R}\right)$ can be expressed as follows [21]:

$$
\left.\begin{array}{rl}
X_{R}= & -\left(1-t_{R}\right)\left(F_{N(P)} \sin \delta_{(P)}+F_{N(\mathrm{~S})} \sin \delta_{(S)}\right) \\
Y_{R}= & -\left(1+a_{H}\right)\left(F_{N(P)} \cos \delta_{(P)}+F_{N(S)} \cos \delta_{(S)}\right) \\
N_{R}= & -\left(x_{R}+a_{H} x_{H}\right)\left(F_{N(P)} \cos \delta_{(P)}+F_{N(S)} \cos \delta_{(S)}\right) \\
& +\left(1-t_{R}\right)\left(y_{R(P)} F_{N(P)} \sin \delta_{(P)}+y_{R(S)} F_{N(S)} \sin \delta_{(S)}\right)
\end{array}\right\}
$$

where $x_{R}$ and $y_{R}$ represent the coordinates of the rudder, respectively. For the reference SWATH ship, $x_{R}=x_{R(P)}=x_{R(S)}$ and $y_{R(P)}=-y_{R(S)} \cdot t_{R}$ is the rudder resistance deduction factor. $a_{H}$ is an increase factor for rudder force, $x_{H}$ is the position of an additional lateral force. In this study, $x_{H}=-0.45 \mathrm{~L}$ according to Yoshimura and Ma (2003) [22]. According to Kijima et al. (1990) [9] and Liu et al. (2017) [10], $t_{R}$ and $a_{H}$ can be estimated as follows:

$$
\begin{aligned}
& t_{R}=-0.28 C_{b}+0.45 \\
& a_{H}=0.627 C_{b}-0.153
\end{aligned}
$$

The $F_{N}$ in Eq. (9) is the rudder normal force and it can be expressed as [13]:

$$
F_{N(P),(S)}=0.5 \rho A_{R}\left(u_{R(P),(S)}^{2}+v_{R(P),(S)}^{2}\right) \frac{6.13 \Lambda_{R}}{\Lambda_{R}+2.25} \sin \alpha_{R(P),(S)}
$$

Here, $\rho$ is the water density, while $A_{R}$ and $\Lambda_{R}$ are the rudder area and aspect ratio, respectively. In this study, $A_{R}=A_{R(P)}=A_{R(S)}$ and $\Lambda_{R}=\Lambda_{R(P)}=\Lambda_{R(S)}$ due to identical geometry of the twin rudders. $\alpha_{R}$ represents the effective inflow angle and can be expressed as shown in the following equations referring to Khanfir et al. (2011) [18,19]:

$$
\begin{aligned}
& \alpha_{R(P),(S)}=\delta_{(P),(S)}-\beta_{R(P),(S)} \gamma_{R(P),(S)}-\tan ^{-1}\left(y_{R(P),(S)} / x_{P(P),(S)}\right) \\
& \beta_{R(P),(S)}=\beta-L_{R(P),(S)}^{\prime} r^{\prime}
\end{aligned}
$$

where $\beta_{R}$ is the drift angle at rudder, $\gamma_{R}$ and $L_{R}^{\prime}$ represent the flow straightening coefficient due to sway velocity and yaw rate. Khanfir et al. (2011) [18] demonstrated that the flow straightening coefficient $L_{R}$ and $\gamma_{R}$ for TPTR ship show a slight asymmetric behavior for port and starboard turning for a TPTR ship. $L_{R}$ is estimated to be nearly as $x_{R}$ for both port 
and starboard rudder. For $\gamma_{R}, \gamma_{R(P)}=0.97$ and $\gamma_{R(S)}=0.98$ for port turning, while $\gamma_{R(P)}=1.15$ and $\gamma_{R(S)}=0.96$ for starboard turning, according to CMT experiment. In this study, $L_{R}^{\prime}=x_{R} / L$ is adopted and $L_{R}^{\prime}=L_{R(P)}^{\prime}=L_{R(S)}^{\prime}$. For simplification, $\gamma_{R}$ is calculated by Yoshimura and Ma (2003) [22] method under the assumption that the $\gamma_{R}$ is symmetry for port and starboard rudders and similar for port and starboard maneuvers. $\gamma_{R}$ is written as:

$$
\gamma_{R}=0.21+1.6 \frac{B}{L} C_{b}
$$

In Eq. (12), $u_{R}$ and $v_{R}$ represent the inflow velocity to rudder, which are expressed as:

$$
\begin{aligned}
& \left.u_{R(P),(S)}=\varepsilon_{(P),(S)} u_{P(P),(S)} \sqrt{\eta_{(P),(S)}\left[1+\kappa_{(P),(S)}\left(\sqrt{1+\frac{8 K_{T(P),(S)}}{\pi J_{P(P),(S)}^{2}}-1}\right)^{2}+\left(1-\eta_{(P),(S)}\right)\right.}\right] \\
& \varepsilon_{(P),(S)}=\left(1-w_{R(P),(S)}\right) /\left(1-w_{P(P),(S)}\right) \\
& u_{P(P),(S)}=\left(1-w_{P(P),(S)}\right)\left(y_{P(P),(S)} r+u\right) \\
& v_{R(P),(S)}=u_{R(P),(S)} \tan \left(\delta_{R(P),(S)}\right)
\end{aligned}
$$

where $\varepsilon$ is a ratio of wake fraction, $u_{P}$ is the longitudinal inflow velocity to the propeller, $\eta$ is the ratio between propeller diameter and rudder span length, $\kappa$ is a constant used to express $u_{R} \cdot \varepsilon$ and $\kappa$ can be estimated by the formulas introduced by Yoshimura and Ma (2003) [22]:

$$
\begin{aligned}
& \varepsilon=0.7+1.9 \frac{B}{L} C_{b} \\
& \kappa=\left(0.55-0.8 \frac{B}{L} C_{b}\right) / \varepsilon
\end{aligned}
$$

\subsection{Added mass and added moment of inertia}

The added mass components are estimated by the Hess Smith panel method [23]. It is assumed moving at constant velocity $\boldsymbol{V}$ in unbounded and calm water with neglect of free surface boundary condition by applying double-body model, the total potential $\phi$ is written as the sum of basic potential $\phi_{0}$ and disturbance potential $\varphi$, i.e., $\phi=\phi_{0}+\varphi$. The relative normal velocity on the boundary is expressed as:

$$
\frac{\partial \varphi}{\partial n}=\boldsymbol{V} \cdot \boldsymbol{n}
$$

where $\boldsymbol{n}$ is the unit outward normal vector on the body surface For a distribution of sources $\sigma(q)$, the disturbance potential at a point $p$ in space is written as: 


$$
\varphi(p)=\iint_{S} \frac{\sigma(q)}{r(p, q)} d S
$$

where $r(p, q)$ is the distance from $p$ to $q, \sigma(q)$ is the source strength. By substituting Eq. (23) into Eq. (22), the integral equation for the source strength distribution can be written as:

$$
\iint_{s} \sigma(q) \frac{\partial}{\partial n_{p}}\left(\frac{1}{r_{p q}}\right) d s_{q}=\boldsymbol{V} \cdot \boldsymbol{n}
$$

The surface $S$ can be discretized into $N$ plane panels, i.e.,

$$
S=\sum_{j=1}^{N} \Delta \mathbf{s}_{j}
$$

A quadrilateral plane $\Delta Q_{j}$ is used to approximate the local surface $\Delta \mathrm{s}_{j}$. It is assumed that hydrodynamic quantities are associated at a control point defined on each panel and they are evenly distributed on the panel. The source strength on each panel is treated as constant, and the term on the left hand of Eq. (24) can be approximated by:

$$
\iint_{s} \sigma(q) \frac{\partial}{\partial n_{p}}\left(\frac{1}{r_{p q}}\right) d s_{q} \approx \sum_{j=1}^{N} \sigma_{j} \iint_{\Delta Q_{j}} \frac{\partial}{\partial n_{p}}\left(\frac{1}{r_{p q}}\right) d s_{q}
$$

The term on the right hand of Eq. (26) can be discretized into the form of a set of linear equations written as:

$$
\begin{aligned}
& \sum_{j=1}^{N} a_{i j} \sigma_{j}=b_{i},(i=1,2 \ldots, N) \\
& a_{i j}=\left\{\begin{array}{l}
\iint_{\Delta Q_{j}} \frac{\partial}{\partial n_{p_{i}}}\left(\frac{1}{r_{p_{i}}}\right) d s_{q} \cdots \cdots \cdots \cdot i \neq j \\
2 \pi \ldots \ldots \ldots \ldots \ldots \ldots \cdots \cdot i=j
\end{array}\right. \\
& b_{i}=\boldsymbol{V} \cdot \boldsymbol{n}
\end{aligned}
$$

The source strength can be obtained by solving Eq. (27) with iteration method, and then the induced potential at the control point of each panel can be determined. Subsequently, the added mass can be calculated with the by the form [24]:

$$
m_{j i}=\rho \iint_{s} \varphi_{i} \frac{\partial \varphi_{j}}{\partial n} d s,(i, j=1,2 \cdots 6)
$$

It should be pointed outed that the hydrodynamic forces and the kinematical parameters in the following text are non-dimensionalized by the speed $U$, water density $\rho$, ship length $L$ and draft $d$. The non-dimensional form is given in Table 1. Symbols with the prime ' stand for non-dimensional value in the following text. 
Table 1 Non-dimensional forms

\begin{tabular}{|c|c|c|c|}
\hline Parameters & Non-dimensionalization & Parameters & Non-dimensionalization \\
\hline$u, v$ & $U$ & $N$ & $0.5 \rho L^{2} d U^{2}$ \\
\hline$r$ & $U / L$ & $m_{x}, m_{y}$ & $0.5 \rho L^{2} d$ \\
\hline$X, Y, F_{N}$ & $0.5 \rho L d U^{2}$ & $J_{z}$ & $0.5 \rho L^{4} d$ \\
\hline
\end{tabular}

\section{Model ship and free running test}

The main particulars of the reference SWATH model are listed in Table 2. The relative centerline distance between the demihulls is defined as $C D_{L}$, where $C D_{L}=C D / L$ and $C D$ is the centerline distance between the demihulls. Table 3 lists the different layouts studied in this paper. It should be noted that the particulars shown in Table 2 are corresponding to the SWATH model with configuration $C D 2$.

Table 2 Main particulars of the SWATH

\begin{tabular}{|c|c|c|}
\hline Particulars & Symbols & Values \\
\hline \multicolumn{3}{|l|}{ Hull } \\
\hline Water line length $(\mathrm{m})$ & $L$ & 3 \\
\hline Beam demi-hull (m) & $b$ & 0.258 \\
\hline Draft $(\mathrm{m})$ & $d$ & 0.25 \\
\hline Displacement overall $\left(\mathrm{m}^{3}\right)$ & $\nabla$ & 0.213 \\
\hline Block coefficient demihull & $C_{b}$ & 0.55 \\
\hline Longitudinal center of gravity from midship, fwd+ (m) & $L C G$ & 0.115 \\
\hline Vertical center of gravity above keel (m) & $V C G$ & 0.342 \\
\hline Radius of gyration for roll (m) & $k_{x x}$ & 0.38 \\
\hline Radius of gyration for pitch (m) & $k_{y y}$ & 0.812 \\
\hline \multicolumn{3}{|l|}{ Propeller } \\
\hline Propeller diameter $(\mathrm{m})$ & $D_{P}$ & 0.133 \\
\hline Number of blades & $Z$ & 4 \\
\hline Pitch ratio & - & 0.85 \\
\hline Longitudinal location (m) & $x_{P}$ & -1.58 \\
\hline Lateral location (m) & $y_{P}$ & \pm 0.395 \\
\hline \multicolumn{3}{|l|}{ Rudder } \\
\hline Rudder area $\left(\mathrm{m}^{2}\right)$ & $A_{R}$ & 0.012 \\
\hline Rudder height (m) & $H_{R}$ & 0.134 \\
\hline Longitudinal location (m) & $x_{R}$ & -1.67 \\
\hline Lateral location (m) & $y_{R}$ & \pm 0.395 \\
\hline
\end{tabular}


Table 3 Different demihull separation layouts of the SWATH

\begin{tabular}{|c|c|c|}
\hline Configuration & $C D_{L}$ & Beam overall $(B / L)$ \\
\hline$C D 1$ & 0.251 & 0.337 \\
\hline$C D 2$ & 0.263 & 0.349 \\
\hline$C D 3$ & 0.279 & 0.365 \\
\hline
\end{tabular}

The free running tests presented in this study are carried out in the Ocean Basin of Harbin Engineering University, as shown in Fig. 2. The basin dimensions are $50 \mathrm{~m}$ long, $30 \mathrm{~m}$ wide and $10 \mathrm{~m}$ deep. The test programs include $35^{\circ}$ starboard turning under $F r=0.202\left(V_{m}=\right.$ $1.1 \mathrm{~m} / \mathrm{s})$ and $F r=0.101\left(V_{m}=0.55 \mathrm{~m} / \mathrm{s}\right)$, and $10^{\circ} / 10^{\circ}$ zigzag maneuver under $F r=0.101$ $\left(V_{m}=0.55 \mathrm{~m} / \mathrm{s}\right)$ in calm water. The Froude number is given by $F r=U / \sqrt{g L}$. The propellers are kept at a constant revolution rate throughout a maneuver. In addition, port side propeller rotates clockwise while starboard side propeller rotates anticlockwise.

Fig. 3 shows the main onboard devices. The two demihulls are connected by six aluminum rods. A propeller and a rudder are installed aft each demi hull. Each propeller is driven by a servo motor. Another two servo motors are used to rotate the rudders to specified angles. The propeller revolutions and rudder angles are measured and recorded during the tests under control of the onboard Micro Controller Unit (MCU) through feedback loop. Pitch, roll, yaw angles and vessel speed are measured by a fiber optical gyro. The yaw rate is computed by differentiating the measured angles. Four lead-acid batteries were used as a power source which supplied the onboard devices such as the gyro, servo motors, data collector, MCU and router. The model trajectory is monitored by the tracking system, which consists of a camera and a LED light. The camera is fixed to a platform $10 \mathrm{~m}$ above the water surface and used to captures the LED light onboard. The horizontal position of the model ship can be estimated from the image analysis. All synchronized signals are wirelessly transmitted to the ground control computer, which manages all the test procedures.

All the onboard devices are included during the adjustment of draft, position of gravity and mass moment of inertia of the model with configuration $C D 2$. Then they are kept concentrated at one location throughout the free running tests. To perform maneuvers with configuration $C D 1$ and $C D 3$, the demihulls are moved symmetrically in y direction. The $L C G$, $V C G$ and lateral mass moment of inertia are the same for each configuration, but the longitudinal mass moment of inertia is different and this variance is not considered in the experiment.
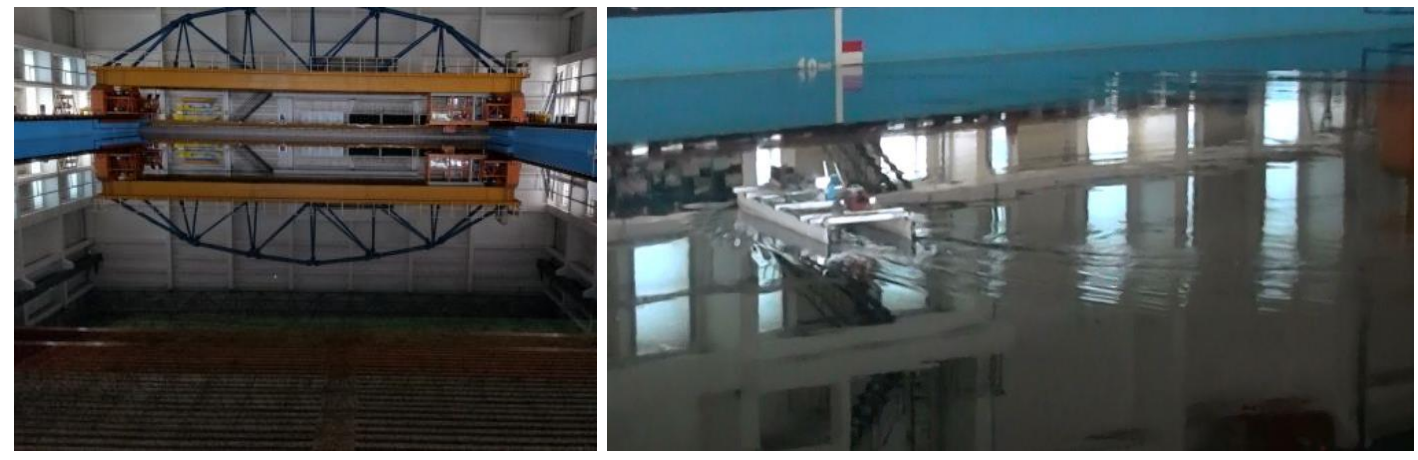

Fig. 2 Free running model tests in Harbin Engineering University 


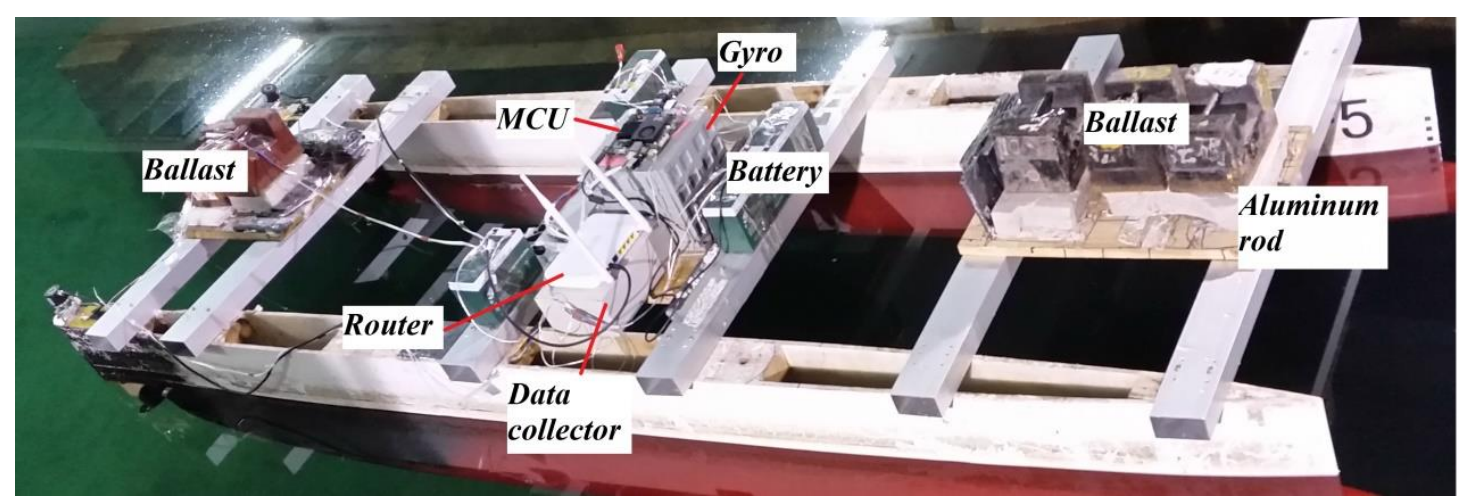

Fig. 3 Model ship setup

\section{Numerical method}

This part describes the numerical method for virtual captive model tests. The RANS solver of the CFD software STAR-CCM+ is used for computations. The SST k- $\omega$ turbulence model is applied to solve governing equations since it performs well in adverse pressure gradients and separated flow. The Finite Volume Method (FVM) is applied to the discretize the fluid domain. The convective and diffusion terms are discretized using second-order upwind scheme. The temporal terms are discretized by adopting first order backward Euler scheme. The Semi-Implicit Method for Pressure-Linked Equations (SIMPLE) algorithm is used to deal with pressure-velocity coupling. The Volume of Fluid (VOF) method is used for the evolution of free surface. The Rotation and Translation module is applied for the simulation of oblique towing tests (OTT) and circular motion tests (CMT).

\subsection{Computational domain and grid}

The computational domain with boundary conditions is shown in Fig. 4. This cuboid domain is set as $1.0 \mathrm{~L}$ in front of the bow, $2.5 \mathrm{~L}$ behind the stern, $1.8 \mathrm{~L}$ in lateral direction, $0.5 \mathrm{~L}$ above and $1.5 \mathrm{~L}$ below the calm water surface. The no-slip wall boundary condition is applied to the hull body. The pressure outlet condition is used for the outlet. The rest of boundaries are treated as velocity inlet. In addition, a numerical wave damping is set on side boundaries in order to eliminate the undesirable reflection of waves.

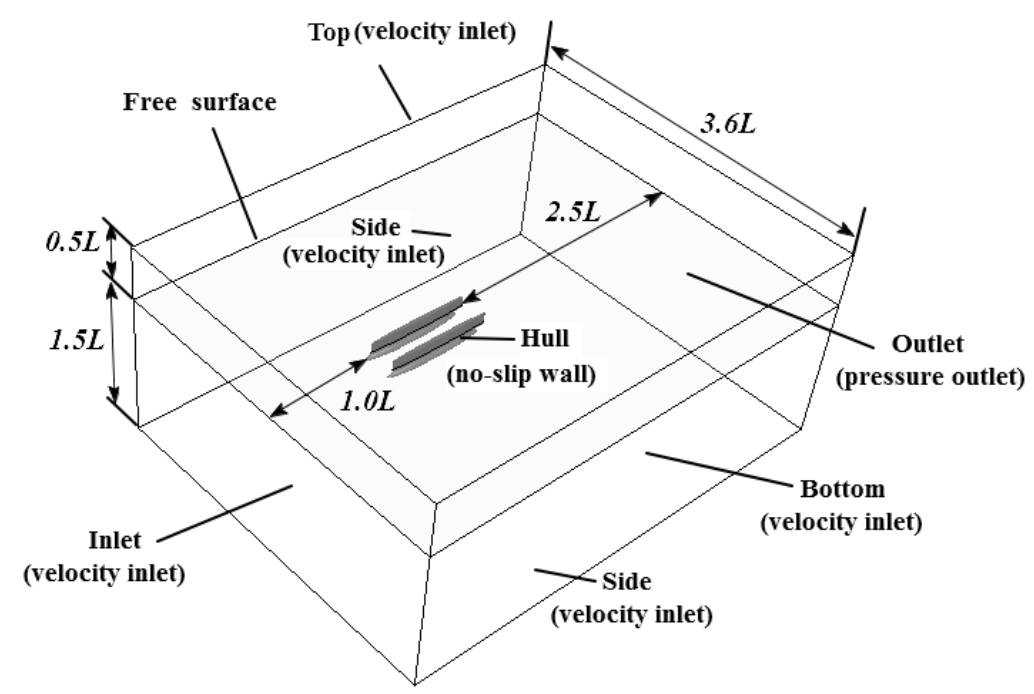

Fig. 4 Computational domain and boundary conditions 
Fig. 5 shows the grid created to discretize the computational domain. Six layers of prismatic cells with a growth ratio of 1.2 are generated to better resolve near-wall flow. The thickness of the first grid layer is set as $0.0011 \mathrm{~m}$ following the recommended nondimensional distance $y+$ ranging from 30 to 100 for the wall functions [25]. Volume refinement blocks are created around the hull, free surface as well as the Kelvin wave area to better resolve the flow filed.
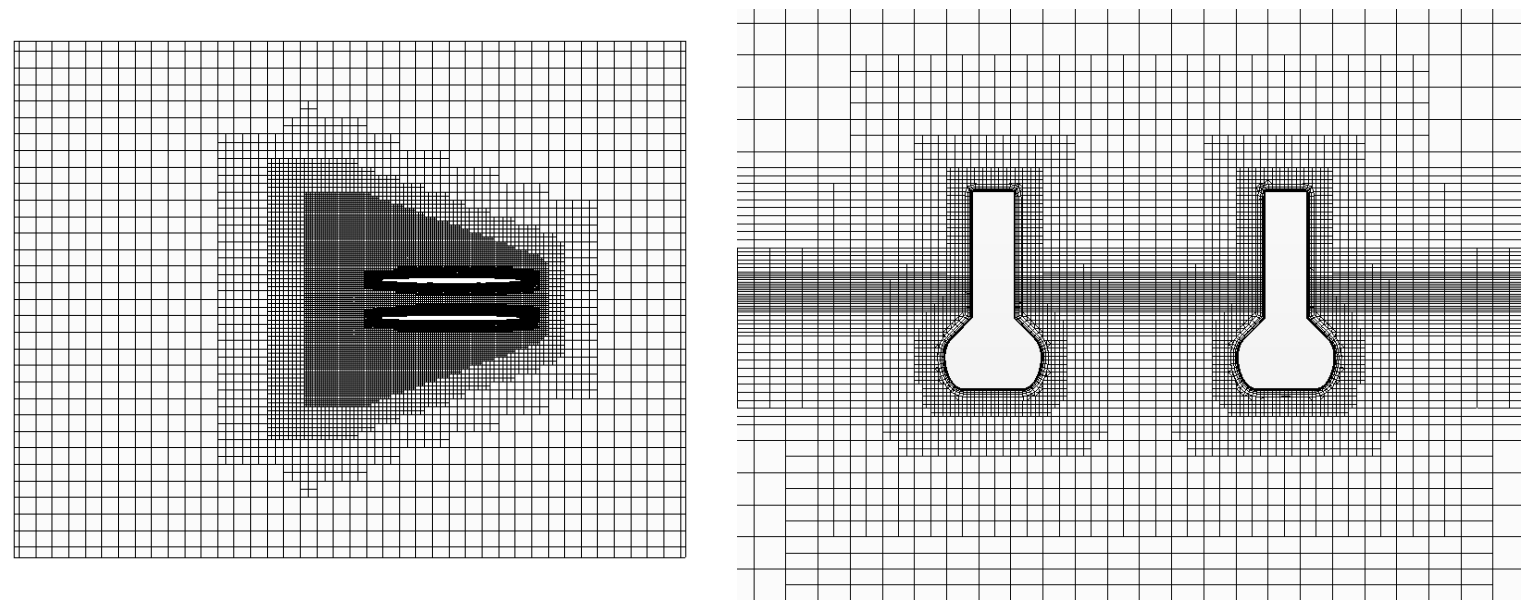

Fig. 5 Computational grid

\subsection{Convergence study}

The convergence study is conducted using the Grid Convergence Index (GCI) method presented by Celik et al. (2008) [26], which is a widely used and recommended method which has been evaluated in a large number of CFD cases. The verification procedure is performed for the configuration $C D 2$, and a steady turn case of $r^{\prime}=0.4$ under $F r=0.202$ is selected to estimate the numerical uncertainties. The forces and moment acting on the hull are investigated.

Three sets of grid spacings and time steps are considered in the study. The grid is constructed referring to a basic size so as to make the grid refined systematically. The coarse, medium and fine grids that are generated based on a constant refinement ratio $r_{G}=\sqrt{2}$ consist of $1.31 \mathrm{M}, 2.58 \mathrm{M}$ and $4.98 \mathrm{M}$ cells, respectively. The coarse, medium and fine time step are selected as $0.0424 \mathrm{~s}, 0.03 \mathrm{~s}$ and $0.0212 \mathrm{~s}$, respectively, by applying a refinement ratio $r_{T}=\sqrt{2}$. The medium time step is used for grid convergence study and time step convergence analysis is conducted with the medium grid.

The apparent order $p_{S}$ of the GCI method can be calculated using the expression:

$$
p_{S}=|\ln | \varepsilon_{S 32} / \varepsilon_{S 21}|| / \ln \left(r_{S}\right)
$$

where $\varepsilon_{S 32}=\phi_{S 3}-\phi_{S 2}, \varepsilon_{S 21}=\phi_{S 2}-\phi_{S 1} \cdot \phi_{S 1}, \phi_{S 2}$ and $\phi_{S 3}$ denote the solutions on fine, medium and coarse grid or time step, respectively. The subscript $S$ denotes the input parameter ( $T$ for time step and $G$ for grid spacing).

The extrapolated value can be calculated from:

$$
\phi_{\mathrm{ext}}^{21}=\left(r_{S}^{p_{S}} \varepsilon_{S 1}-\varepsilon_{S 2}\right) /\left(r_{S}^{p_{S}}-1\right)
$$


The approximate relative error is expressed as:

$$
e_{a}^{21}=\left|\frac{\phi_{S 1}-\phi_{S 1}}{\phi_{S 1}}\right|
$$

The extrapolated relative error is expressed as:

$$
e_{\mathrm{ext}}^{21}=\left|\frac{\phi_{\mathrm{ext}}^{12}-\phi_{S 1}}{\phi_{\mathrm{ext}}^{12}}\right|
$$

The fine input parameter convergence index is calculated by:

$$
\mathrm{GCI}_{\text {fine }}^{21}=\frac{1.25 e_{a}^{21}}{r_{S}^{p_{S}}-1}
$$

Table 4 and Table 5 show the results of convergence study. The maximum numerical uncertainty among $X^{\prime}, Y^{\prime}, N^{\prime}$ is less than $2 \%$ for the grid convergence study as presented in Table 4. As for the time step convergence, the maximum numerical uncertainty is less than $5 \%$ as shown in Table 5. It can be said that a very low level of uncertainty is estimated for the longitudinal and lateral forces and yaw moment. Thus, in order to balance the computation cost and accuracy, the medium grid with $2.58 \mathrm{M}$ cells and medium time step of $0.03 \mathrm{~s}$ are used in the following numerical calculations.

Table 4 Results of grid convergence study

\begin{tabular}{|c|c|c|c|c|c|c|c|c|}
\hline Items & $\phi_{G 1}$ & $\phi_{G 2}$ & $\phi_{G 3}$ & $p_{G}$ & $\phi_{\mathrm{ext}}^{21}$ & $e_{a}^{21}(\%)$ & $e_{\mathrm{ext}}^{21}(\%)$ & $\mathrm{GCI}_{\mathrm{fine}}^{21}(\%)$ \\
\hline$X^{\prime}$ & -0.02317 & -0.02346 & -0.0240 & 1.832 & -0.023 & 1.232 & 1.409 & 1.737 \\
\hline$Y^{\prime}$ & 0.04843 & 0.04903 & 0.04843 & 4.160 & 0.048 & 1.256 & 0.390 & 0.486 \\
\hline$N^{\prime}$ & -0.02763 & -0.02763 & 0.05161 & 4.636 & -0.028 & 0.658 & 0.165 & 0.206 \\
\hline
\end{tabular}

Table 5 Results of time step convergence study

\begin{tabular}{|c|c|c|c|c|c|c|c|c|}
\hline Items & $\phi_{T 1}$ & $\phi_{T 2}$ & $\phi_{T 3}$ & $p_{T}$ & $\phi_{\mathrm{ext}}^{21}$ & $e_{a}^{21}(\%)$ & $e_{\mathrm{ext}}^{21}(\%)$ & $\mathrm{GCI}_{\text {fine }}^{21}(\%)$ \\
\hline$X^{\prime}$ & -0.02344 & -0.02346 & -0.02349 & 0.479 & -0.023 & 0.104 & 0.579 & 0.719 \\
\hline$Y^{\prime}$ & 0.04958 & 0.04903 & 0.04791 & 2.082 & 0.050 & 1.099 & 1.028 & 1.298 \\
\hline$N^{\prime}$ & -0.03041 & -0.02992 & -0.02921 & 1.128 & -0.031 & 1.593 & 3.223 & 4.163 \\
\hline
\end{tabular}

\section{Results and discussion}

\subsection{Hydrodynamic derivatives}

In this study, the ship hull under consideration is the SWATH model of layout CD2 without propellers and rudders. Simulations of CMT and OTT are carried out and hull forces and moment are obtained. The computation cases are summarized in Table 6. All the computations are performed under $F r=0.202$ in calm water condition. The freedom of trim and sinkage are restricted during the simulations. 
Table 6 Computation cases for OTT and CMT

\begin{tabular}{|c|c|c|c|}
\hline Tests & $F r$ & $r^{\prime}$ & $\beta\left(^{\circ}\right)$ \\
\hline OTT & 0.202 & 0 & $0, \pm 4, \pm 8, \pm 12, \pm 16, \pm 20$ \\
\hline CMT & 0.202 & $-0.4,0.2,0.4,0.6$ & $0, \pm 4, \pm 8, \pm 12, \pm 16, \pm 20$ \\
\hline
\end{tabular}

Fig. 6 shows the results of hydrodynamic forces and moment acting on each demihulls and the entire ship model in steady turns simulation with $r^{\prime}=-0.4$ and $r^{\prime}=0.4$. Subscripts $(\mathrm{PH})$ and $(\mathrm{SH})$ indicate demihull on port and starboard side, respectively. From Fig. 6, it can be seen that the forces and moment on port and starboard demihulls with positive $r^{\prime}$ and $\beta$ are almost the same with that on starboard and port demihulls with negative $r^{\prime}$ and $\beta$, respectively. As a result of that, the resultant forces under positive $r^{\prime}$ and $\beta$ shows little difference with that under negative $r^{\prime}$ and $\beta$. Fig. 7 presents the free surface wave pattern of port turning with $r^{\prime}=-0.4, \beta=20^{\circ}$ and starboard turning with $r^{\prime}=0.4, \beta=-20^{\circ}$. The flow flied of the two cases shows similarity. Therefore, it can be concluded that the results of simulation are symmetric for steady port and starboard turning.

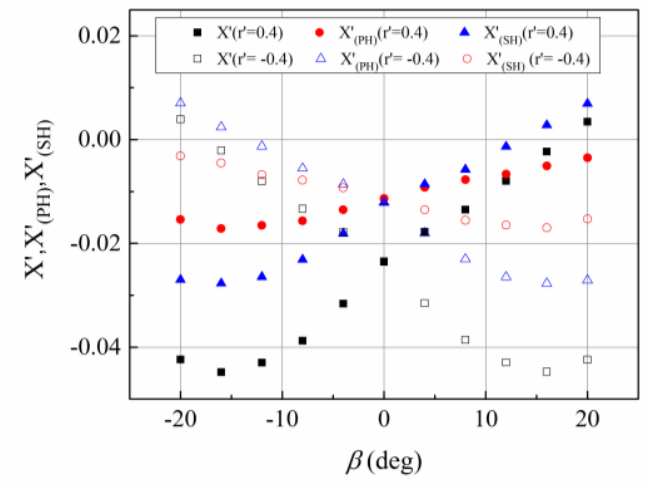

(a) Longitudinal force

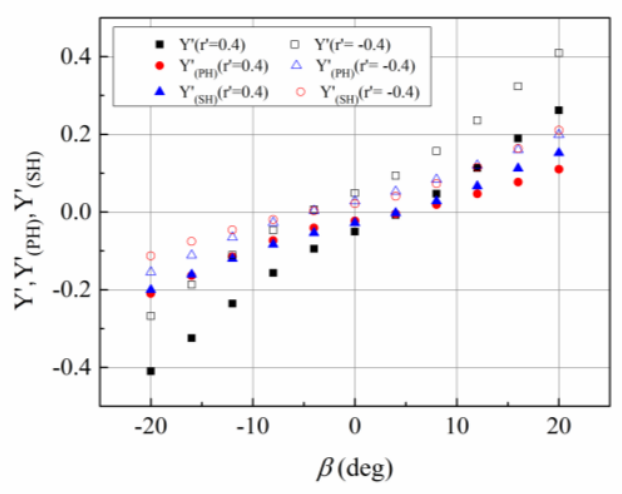

(b) Lateral force

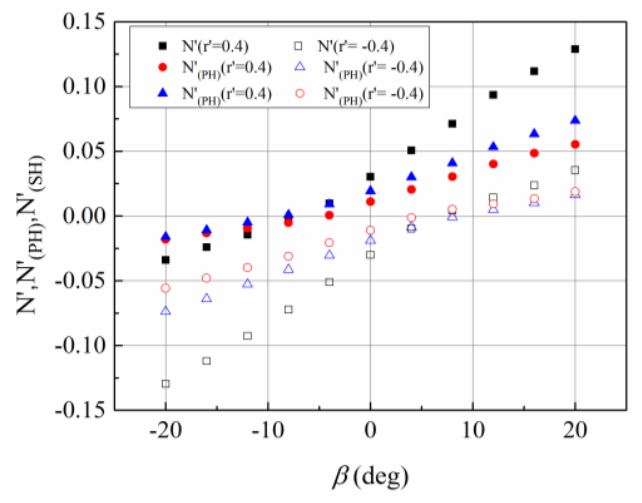

(c) Yaw moment

Fig. 6 Hydrodynamic forces and moment in steady port and starboard turning 


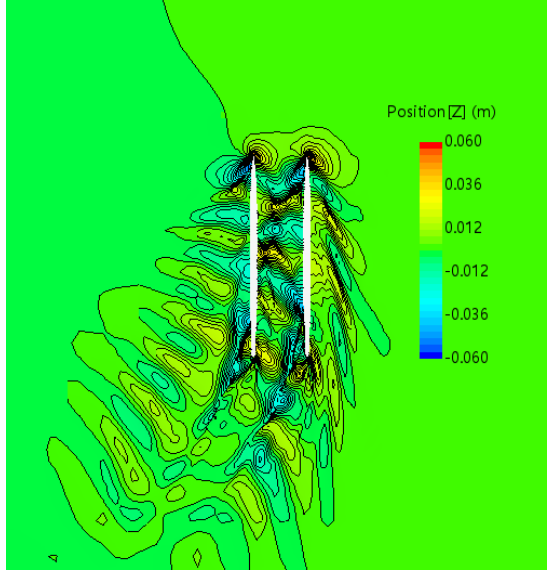

(a) $r^{\prime}=-0.4, \beta=20^{\circ}$

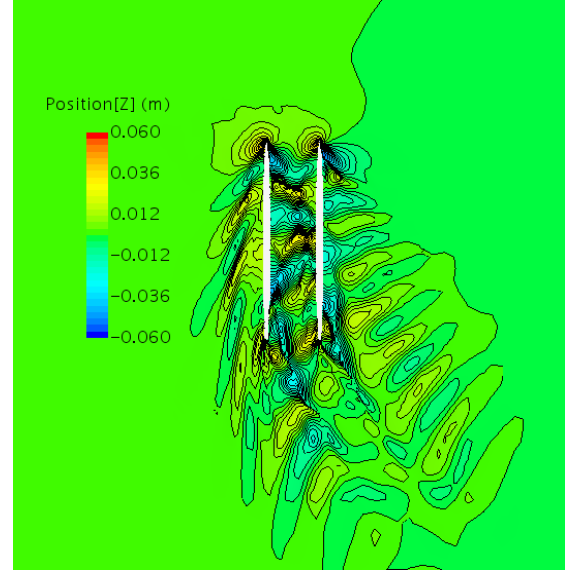

(b) $r^{\prime}=0.4, \beta=-20^{\circ}$

Fig. 7 Free surface wave pattern

Fig. 8 shows the computed longitudinal force, lateral force and yaw moment of CMT and OTT simulations. With the results of that, hydrodynamic derivatives are determined by a least square method using the obtained data based on Eq. (1)-(2). It should be noted that the added mass components in the equations of motion can not be obtained from the present CFD. They are estimated by the method introduced in Section 2.5. The results of hydrodynamic derivatives and added mass components are listed in Table 7 . The fitting curves with the generated hydrodynamic derivatives are plotted with dash in Fig. 9, together with the CFD results shown as symbols. In the view of practice, the fitting accuracy is sufficient.

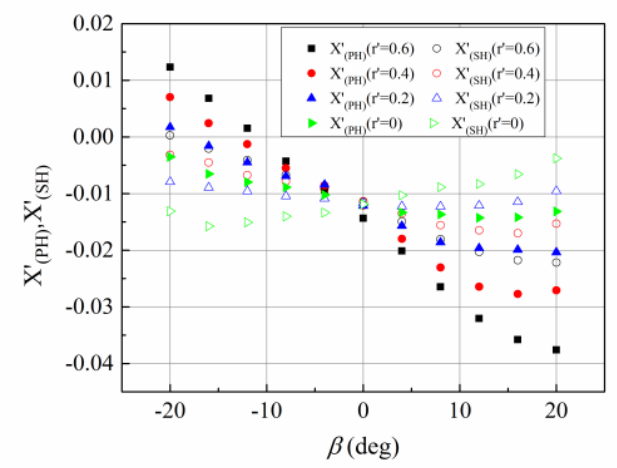

(a) Longitudinal force

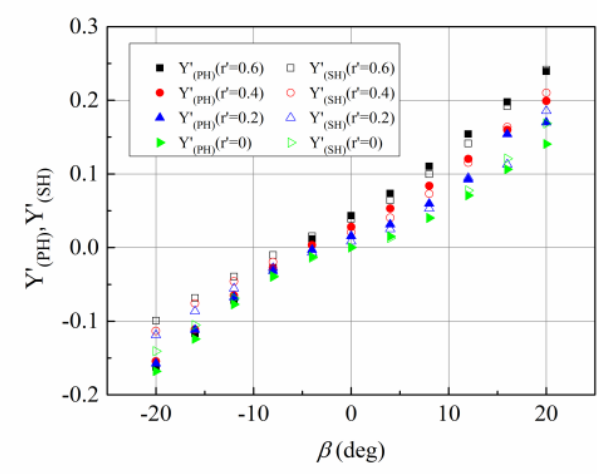

(b) Lateral force

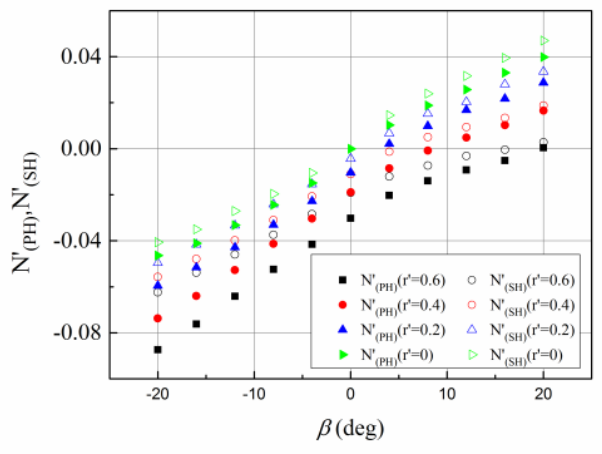

(c) Yaw moment

Fig. 8 OTT and CMT results 
Table 7 Hydrodynamic derivatives and added mass components

\begin{tabular}{|c|c|c|c|c|c|}
\hline Items & Values & Items & Values & Items & Values \\
\hline$X_{v v}^{\prime}$ & 0.00048 & $Y_{v}^{\prime}$ & -0.588 & $N_{v}^{\prime}$ & -0.303 \\
\hline$X_{r r}^{\prime}$ & -0.315 & $Y_{r}^{\prime}$ & 0.125 & $N_{r}^{\prime}$ & -0.0721 \\
\hline$X_{v r}^{\prime}$ & -0.0115 & $Y_{v v v}^{\prime}$ & -2.702 & $N_{v v v}^{\prime}$ & 0.412 \\
\hline$X_{v v v v}^{\prime}$ & 0.403 & $Y_{v v r}^{\prime}$ & 0.574 & $N_{v v r}^{\prime}$ & -0.393 \\
\hline$m_{x}^{\prime}$ & 0.00625 & $Y_{v r r}^{\prime}$ & -0.669 & $N_{v r r}^{\prime}$ & 0.129 \\
\hline$m_{y}^{\prime}$ & 0.503 & $Y_{r r r}^{\prime}$ & 0.0383 & $N_{r r r}^{\prime}$ & -0.0328 \\
\hline$J_{z}^{\prime}$ & 0.0284 & $R_{0}^{\prime}$ & 0.0237 & & \\
\hline
\end{tabular}
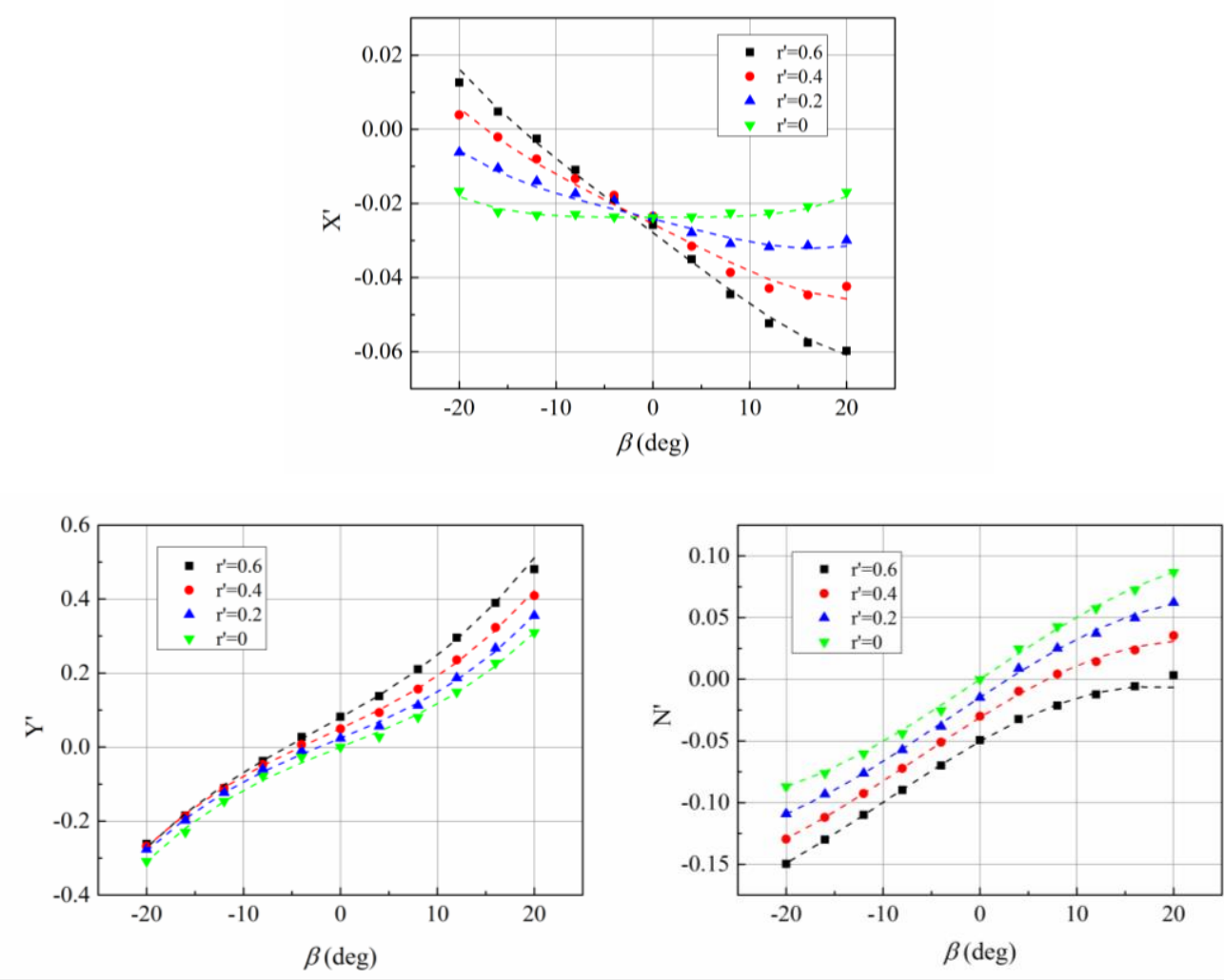

Fig. 9 Analysis results of forces and moment

\subsection{Simulations of maneuvers}

With the interactive coefficients of hull-propeller-rudder from the empirical method and hydrodynamic derivatives generated by virtual captive model tests, maneuvering simulations are performed for the SWATH model of layout $C D 2$ using the 3-DOF MMG mathematical model. Turning with $\delta=35^{\circ}$ under $F r=0.202$ and $F r=0.101$, and $10^{\circ} / 10^{\circ}$ zigzag maneuver under $F r=0.101$ are considered. Propeller revolution remains a constant during a maneuver simulation. The propeller open water performance results are shown in Fig. 10. Parameters for representing the propeller thrust coefficient are: $\left(k_{0}, k_{1}, k_{2}\right)=(0.4136,-0.4003,-0.0924)$. The hydrodynamic derivatives for estimation of hull forces and moment are listed in Table 7 along with added mass components. The remaining parameters used in the simulations for propeller and rudder modules are shown in Table 8. 


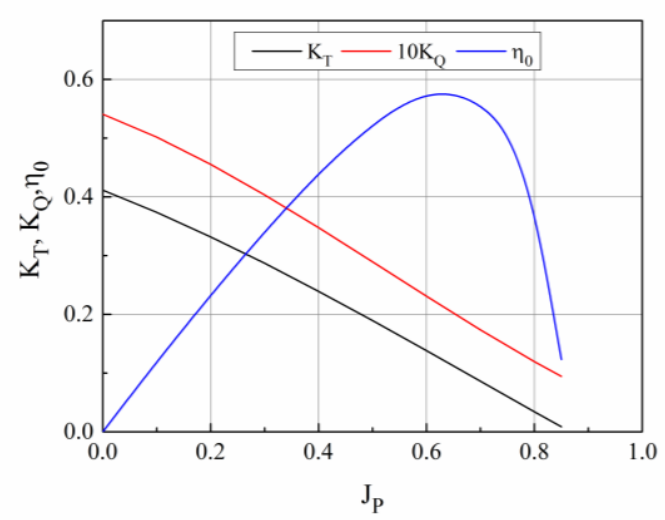

Fig.10 Open water data of propeller

Table 8 Parameters for propeller and rudder modules

\begin{tabular}{|c|c|c|c|c|c|}
\hline Items & Values & Items & Values & Items & Values \\
\hline$t_{P}$ & 0.2 & $w_{P 0}$ & 0.225 & $t_{R}$ & 0.296 \\
\hline$a_{H}$ & 0.192 & $\Lambda_{R}$ & 1.48 & $\varepsilon$ & 1.065 \\
\hline$\kappa$ & 0.372 & $\gamma_{R}$ & 0.517 & $\eta$ & 0.993 \\
\hline$x_{H}^{\prime}$ & -0.45 & & & & \\
\hline
\end{tabular}

The predicted and tested trajectories, speed and yaw rate in $35^{\circ}$ turning maneuver are presented in Fig. 11-Fig. 13, and a comparison of turning indices of advance, transfer, tactical diameter and radius are given in Table 8. As shown in Fig. 11, the numerical prediction method overestimates the tactical diameter and radius with respect to the free running data under the two approach speeds. The comparison errors of tactical diameter and radius are $-11.33 \%$ and $-14.92 \%$ for $F r=0.202,-9.96 \%$ and $-8.33 \%$ for $F r=0.101$, respectively. As shown in Fig. 12 and Fig. 13, the overall trends of speed and yaw rate under $F r=0.202$ and $F r=0.101$ are both reasonably matched by simulations. The speed is slightly overestimated during the whole turning maneuver, while the yaw rate is overestimated in the unsteady stage of turning and then underestimated when the steady stage of turning is reached, leading to an underestimation of advance and overestimation of transfer. The comparison errors of advance and transfer are $3.08 \%$ and $-13.98 \%$ for $F r=0.202,-4.27 \%$ and $-8.08 \%$ for $F r=0.101$, respectively. Overall, it is shown that the present method gives satisfactory predictions of turning trajectory and corresponding motion parameters.

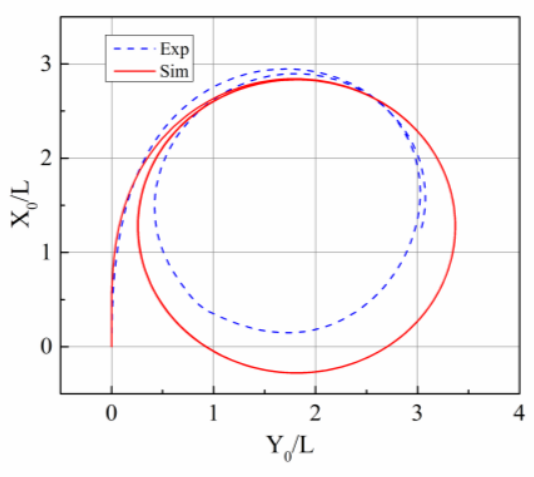

(a) $F r=0.202$

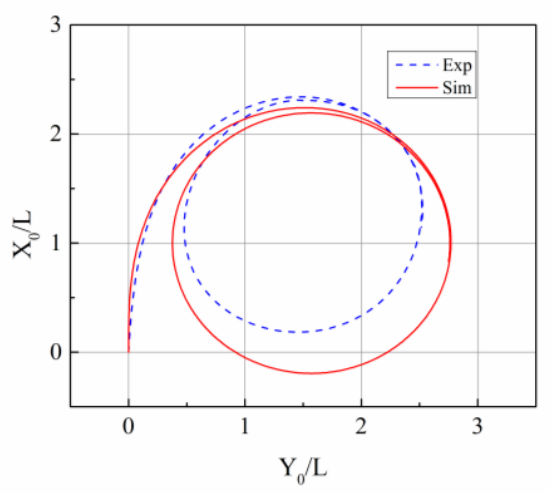

(b) $F r=0.101$

Fig. 11 Comparison of trajectories in the $35^{\circ}$ turning maneuver 


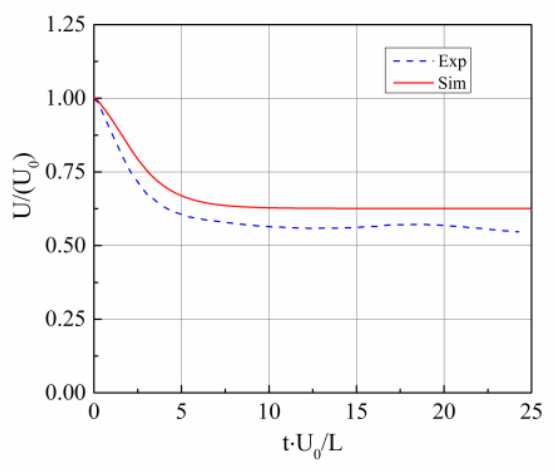

(a) $F r=0.202$

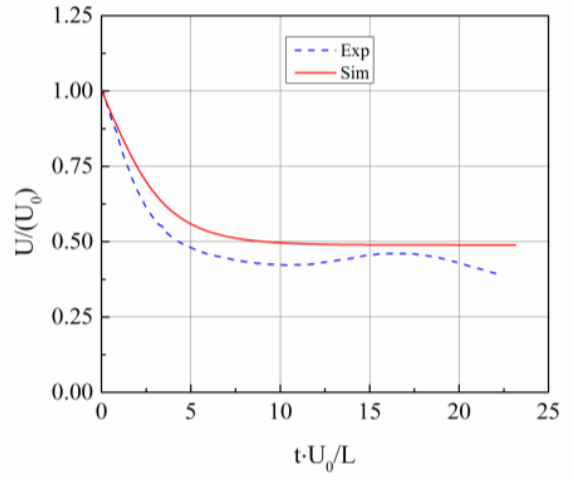

(b) $F r=0.101$

Fig. 12 Comparison of speed in the $35^{\circ}$ turning maneuver

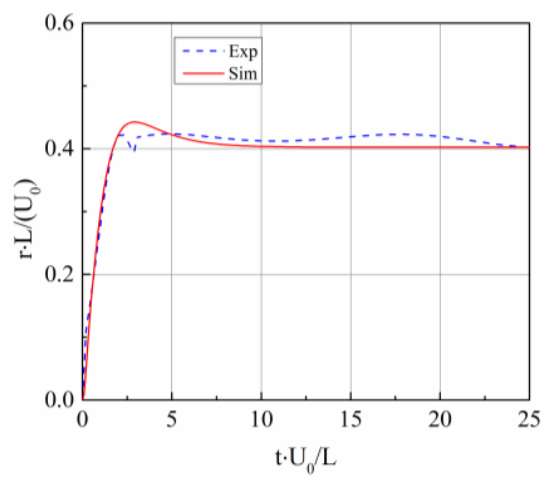

(a) $F r=0.202$

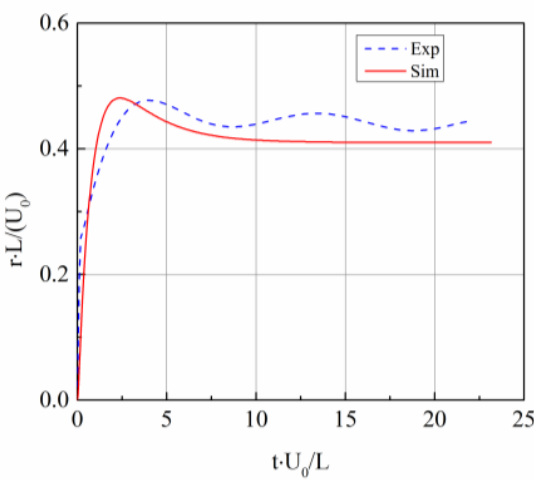

(b) $F r=0.101$

Fig. 13 Comparison of yaw rate in the $35^{\circ}$ turning maneuver

The time history of simulated propeller thrust is presented in Fig. 14. An obvious difference between thrusts from port and starboard propellers can be found. The difference is generated by the lateral locations of port and starboard propellers, according to Eq. (5). The port thrust is about $13 \%$ and $18 \%$ smaller than the starboard thrust under steady turning condition for $F r=0.202$ and $F r=0.101$, respectively. The time history of simulated rudder lateral force is presented in Fig. 15. Similarly, there is a significant difference between lateral forces acting on port and starboard rudders, which is caused by the lateral locations of port and starboard rudders according to Eq. (13). The starboard lateral force is about $60 \%$ smaller than the port lateral force under steady turning condition for both speeds.

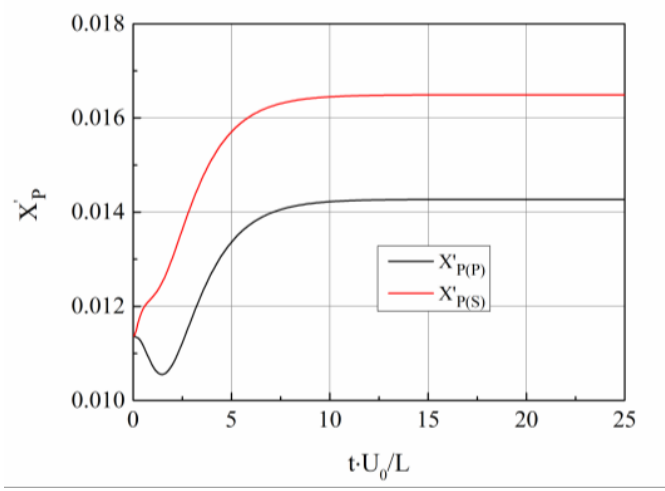

(a) $F r=0.202$

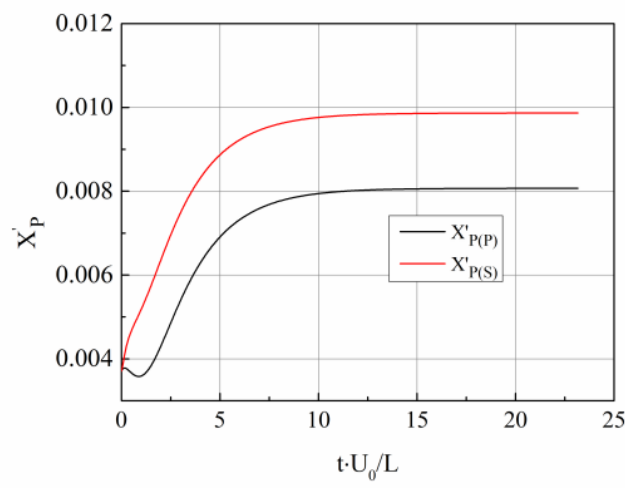

(b) $F r=0.101$

Fig. 14 Simulated propeller thrust in the $35^{\circ}$ turning maneuver 


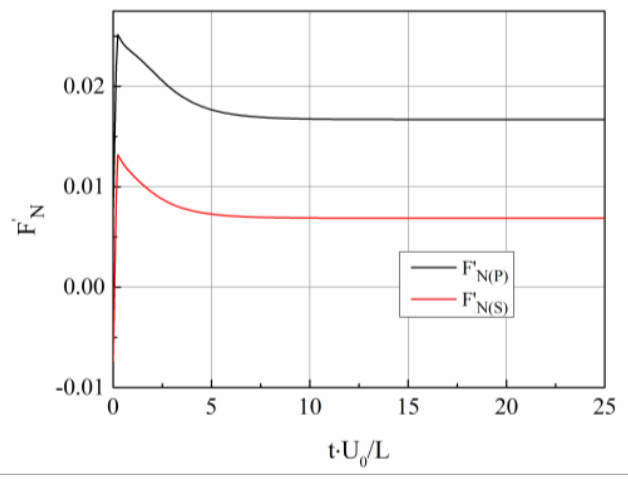

(a) $F r=0.202$

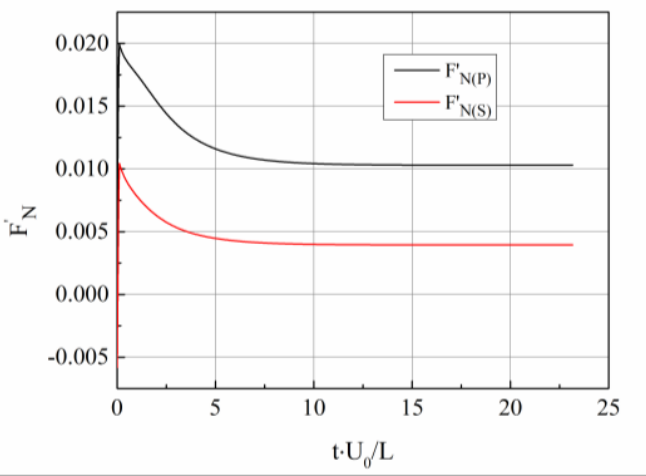

(b) $F r=0.101$

Fig. 15 Simulated rudder normal force in the $35^{\circ}$ turning maneuver

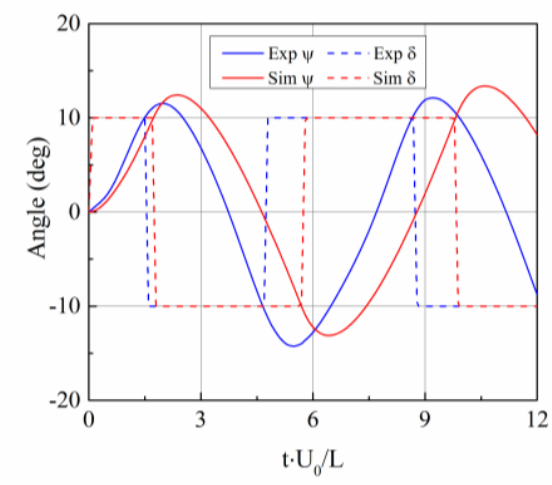

(a) Rudder angle and heading angle

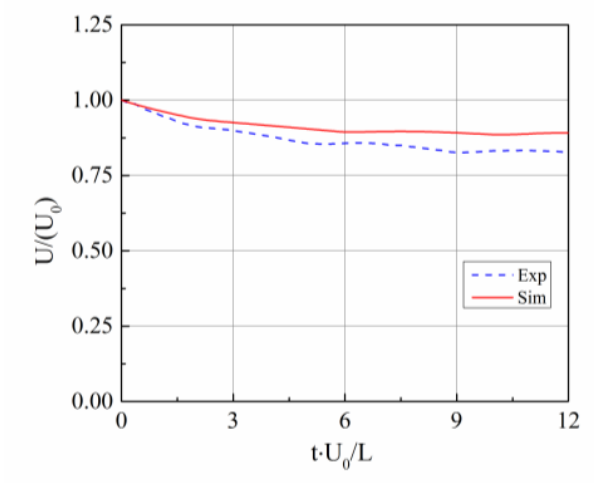

(b) Speed

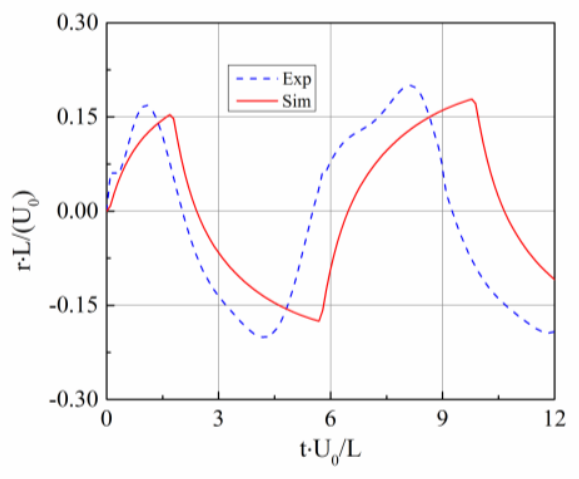

(c) Yaw rate

Fig. 16 Comparison of time histories of motion parameters in $10^{\circ} / 10^{\circ}$ zigzag maneuver $(\mathrm{Fr}=0.101)$

The comparison of predicted results with the model test data of $10^{\circ} / 10^{\circ}$ zigzag maneuver is plotted in Fig. 16, where the rudder angle, heading angle, speed and yaw rate are shown. The time history of corresponding simulated propeller thrust and rudder normal force are presented in Fig. 17. The overshoot angles obtained from experiment and simulation are compared in Table 9. As shown in Fig. 16(a), the predicted result presents a time delay when maximum heading angles are reached. The 1st overshoot angle and 2nd overshoot angle are estimated $0.89^{\circ}$ larger and $1.24^{\circ}$ smaller than that of free running data, respectively. As shown in Fig. 16(b)-(c), the yaw rate and speed from simulation present similar trend with the experimental results. The speed is overestimated during the zigzag maneuver, while the yaw rate shows periodic changes and its peak values are underestimated. On the whole, the present method provides a reasonable prediction of zigzag maneuver for the SWATH. 


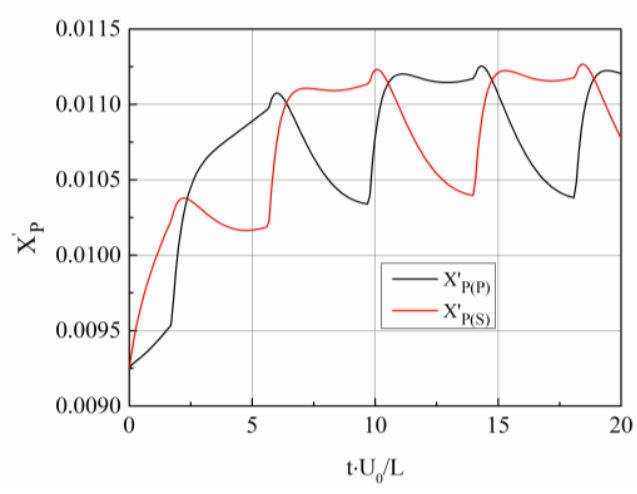

(a) Propeller thrust

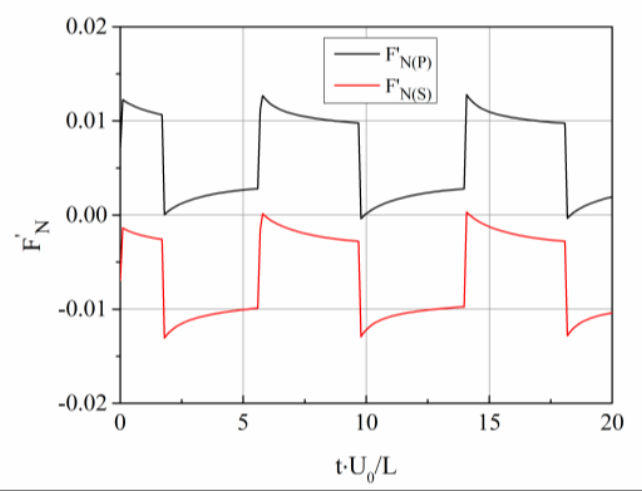

(b) Rudder normal force

Fig. 17 Simulated propeller and rudder forces in the $10^{\circ} / 10^{\circ}$ zigzag maneuver $(\mathrm{Fr}=0.101)$

Table 9 Comparison of predicted maneuverability parameters with free running data

\begin{tabular}{|c|c|c|c|c|}
\hline Free maneuvers & Parameters & Exp & Sim & Difference (\%) \\
\hline \multirow{3}{*}{$\begin{array}{c}35^{\circ} \text { turning } \\
(F r=0.202)\end{array}$} & Advance(L) & 2.92 & 2.83 & 3.08 \\
\cline { 2 - 5 } & Transfer(L) & 1.43 & 1.63 & -13.98 \\
\cline { 2 - 5 } & Tactical diameter(L) & 3.0 & 3.34 & -11.33 \\
\cline { 2 - 5 } & Radius(L) & 1.34 & 1.54 & -14.92 \\
\hline \multirow{3}{*}{$\begin{array}{c}35^{\circ} \text { turning } \\
(F r=0.101)\end{array}$} & Advance(L) & 2.34 & 2.24 & 4.27 \\
\cline { 2 - 5 } & Transfer(L) & 1.36 & 1.47 & -8.08 \\
\cline { 2 - 5 } & Tactical diameter $(\mathrm{L})$ & 2.51 & 2.76 & -9.96 \\
\cline { 2 - 5 } & Radius $(\mathrm{L})$ & 1.08 & 1.17 & -8.33 \\
\hline \multirow{2}{*}{$\begin{array}{c}10^{\circ} / 10^{\circ} \text { zigzag } \\
(F r=0.101)\end{array}$} & 1st overshoot angle $\left({ }^{\circ}\right)$ & 1.53 & 2.37 & -54.9 \\
\cline { 2 - 5 } & 2nd overshoot angle $\left({ }^{\circ}\right)$ & 4.26 & 3.02 & 29.11 \\
\hline
\end{tabular}

\subsection{Experimental results of different demihull separation layouts}

In order to investigate the influence of the lateral location of the demihulls on maneuvering performance of the SWATH, free running model tests of turning with $\delta=35^{\circ}$ under $F r=0.202$ and $10^{\circ} / 10^{\circ}$ zigzag maneuvers under $F r=0.101$ are carried out for three demihull separation layouts (listed in Table 2). The experimental results of turning and zigzag maneuvers for different demihull separation layouts are shown in Fig. 18, and turning indices and overshoot angles are summarized in Table 10. It can be seen from Fig. 18(a) that turning trajectories for layout $C D 1$ and $C D 3$ are very similar, while turning trajectory for layout $C D 2$ shows a larger circle. The advances for the three layouts are almost the same, but the tactical diameter and radius for layout $C D 2$ are about $30 \%$ and $20 \%$ larger comparing with the other two layouts. This means that intermediate demihull separation of the SWATH shows worse turning performance. As shown in Fig. 18(b), the time-changing heading angle and rudder angle for the three demihull separation layouts present slight differences during the zigzag maneuver. Maximum differences of overshoot angles among the three layouts are about $1^{\circ}$. It can be said that demihull separation has little influence on the course stability. 


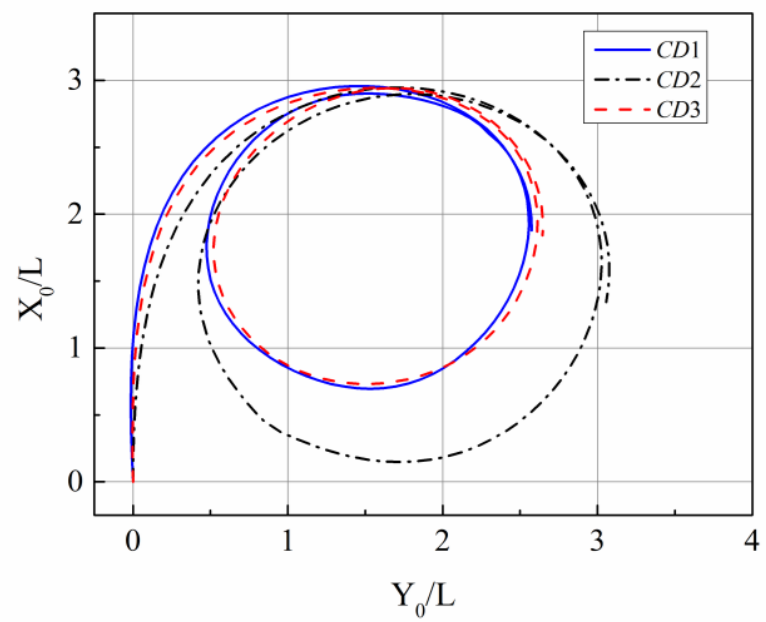

(a)

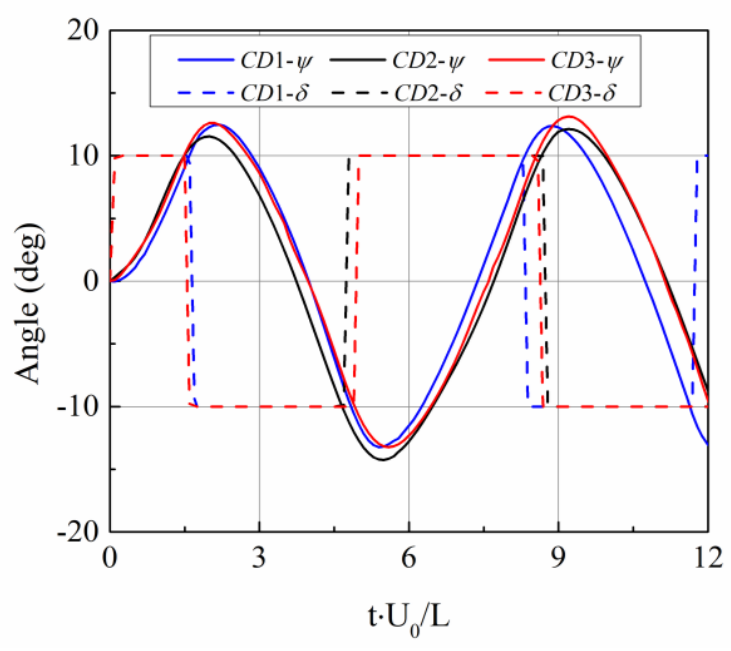

(b)

Fig. 18 Experimental results for different demihull separation layouts: (a) trajectories of $35^{\circ}$ turning; (b) time histories of heading angle and rudder angle in $10^{\circ} / 10^{\circ}$ zigzag maneuvers

Table 10 Maneuverability parameters for different demihull separation layouts

\begin{tabular}{|c|c|c|c|c|}
\hline Free maneuvers & Parameters & $C D 1$ & $C D 2$ & $C D 3$ \\
\hline \multirow{3}{*}{$\begin{array}{c}35^{\circ} \text { turning } \\
(F r=0.202)\end{array}$} & Advance $(\mathrm{L})$ & 2.9 & 2.92 & 2.89 \\
\cline { 2 - 5 } & Transfer(L) & 1.07 & 1.43 & 1.21 \\
\cline { 2 - 5 } & Tactical diameter $(\mathrm{L})$ & 1.05 & 1.34 & 1.06 \\
\cline { 2 - 5 } & Radius $(\mathrm{L})$ & 2.51 & 3 & 2.57 \\
\hline $10^{\circ} / 10^{\circ}$ zigzag & 1 st overshoot angle $\left(^{\circ}\right)$ & 2.47 & 1.53 & 2.61 \\
\cline { 2 - 5 }$(F r=0.101)$ & 2 nd overshoot angle $\left({ }^{\circ}\right)$ & 3.23 & 4.26 & 3.23 \\
\hline
\end{tabular}

\section{Conclusions}

A modular MMG mathematical model is implemented for maneuvering performance simulation of a SWATH ship in this paper. Publicly available empirical methods are selected to estimate the interaction coefficients among the hull, rudder and propeller, while the hydrodynamic derivatives are acquired from simulations of captive model tests using a RANS solver. The convergence study is conducted to check the influence of grid and time step on the virtual captive model tests. Then, a set of captive model tests are simulated to generate hydrodynamic derivatives in the mathematical model.

The $35^{\circ}$ turning and $10^{\circ} / 10^{\circ}$ zigzag maneuvers are simulated with the obtained hydrodynamic coefficients from virtual captive model tests and empirical formulas. The computed results show a good agreement with the free running data. Meanwhile, the numerical method also shows satisfactory prediction in the typical maneuverability parameters. At the practical level, the method presented in this study shown promising applicability in prediction of maneuvering performance of SWATH ship. Moreover, free running model tests have been performed for three demihull separation configurations. The experimental results show that turning performance become worse for the intermediate demihull separation, while the demihull separation shows little impact on zigzag maneuver . Further research is needed to study the hydrodynamic interaction among hull, propeller and rudder of the SWATH in order to improve maneuvering prediction. The virtual captive model 
tests based on CFD can be extended to get the hull-propeller-rudder interaction coefficients in future study.

\section{ACKNOWLEDGEMENT}

This work was supported by the National Natural Science Foundation of China (Grant No. 51979157) and the Natural Science Foundation of Shanghai, China (Grant No. 19ZR1422500).

\section{REFERENCES}

[1] Dubrovsky, V.A., 2010. Multi-hulls: new options and scientific developments. Ships and Offshore Structures, 5(1), 81-92. https://doi.org/10.1080/17445300903149038

[2] Beena, V.I., Subramanian V.A., 2003. Parametric studies on seaworthiness of SWATH ships. Ocean Engineering, 30 (9), 1077-1106. https://doi.org/10.1016/S0029-8018(02)00101-4

[3] Dubrovsky, V.A., Matveevb, K.I., 2006. Small waterplane area ship models: Re-analysis of test results based on scale effect and form drag. Ocean Engineering, 33 (7), 950-963. https://doi.org/10.1016/j.oceaneng.2005.07.006

[4] Qian, P., Yi, H., Li, Y.H., 2015. Numerical and experimental studies on hydrodynamic performance of a small-waterplane-area-twin-hull (SWATH) vehicle with inclined struts. Ocean Engineering, 96 (1), 181191. https://doi.org/10.1016/j.oceaneng.2014.12.039

[5] Guan, G., Yang, Q., Wang, Y. et al., 2021. Parametric design and optimization of SWATH for reduced resistance based on evolutionary algorithm. Journal of Marine Science and Technology, 26, 54-70. https://doi.org/10.1007/s00773-020-00721-w

[6] Abkowitz, M.A., 1964. Lectures on Ship Hydrodynamics-Steering and Manoeuvrability. In: Hydro- and Aerodynamics Lab, Report No. Hy-5, Lyngby, Copenhagen, Denmark.

[7] Yasukawa H., Yoshimura, Y., 2015. Introduction of MMG Standard Method for Ship Maneuvering Predictions. Journal of Marine Science and Technology, 20(1), 37-52. https://doi.org/10.1007/s00773014-0293-y

[8] Hajizadeh, S., Seif, M.S., Mehdigholi, H., 2016. Evaluation of planing craft maneuverability using mathematical modelling. Brodogradnja, 67(1), 85-100.

[9] Kijima, K., Katsuno, T., Nakiri, Y., Furukawa, Y., 1990. On the manoeuvring performance of a ship with the parameter of loading condition. Journal of the Society of Naval Architects of Japan, 168, 141-148. https://doi.org/10.2534/jjasnaoe1968.1990.168_141

[10] Liu, J., Hekkenberg, R., Quadvlieg, F., Hopman, H., Zhao, B.Q., 2017. An integrated empirical manoeuvring model for inland vessels. Ocean Engineering, 137(1), 287-308. https://doi.org/10.1016/j.oceaneng.2017.04.008

[11] He, S., Kellett, P., Yuan, Z. M.,Incecik,A., Turan, O., Boulougouris, E., 2016. Manoeuvring prediction based on CFD generated derivatives. Journal of Hydrodynamics, Series B, 28(2), 284-292. https://doi.org/10.1016/S1001-6058(16)60630-3

[12] Liu, Y., Zou, L., Zou, Z.J., Guo, H.P., 2018. Predictions of ship maneuverability based on virtual captive model tests. Engineering Applications of Computational Fluid Mechanics, 12(1), 334-353. https://doi.org/10.1080/19942060.2018.1439773

[13] Sukas, O.F., Kinaci, O.K., Bal, S., 2019. System-based prediction of maneuvering performance of twinpropeller and twin-rudder ship using a modular mathematical model. Applied Ocean Research, 84, 145162. https://doi.org/10.1016/j.apor.2019.01.008

[14] Sakamoto, N., Ohashi, K., Araki, M., Kume, K.I, Kobayashi H., 2019. Identification of KVLCC2 manoeuvring parameters for a modular-type mathematical model by RaNS method with an overset approach. Ocean Engineering, 188:106257. https://doi.org/10.1016/j.oceaneng.2019.106257

[15] Ardeshiri, S., Mousavizadegan, H., Kheradmand, S., 2020. Virtual simulation of PMM tests independent of test parameters. Brodogradnja, 71(2), 55-73. http://dx.doi.org/10.21278/brod71204

[16] Ogawa, A., Koyama, T., Kijima, K., 1977. MMG Reports - I, Bulletin of the Society of Naval Architects of Japan, No. 575. 
[17] Kulczyk, J., Tabaczek, T., 2014. Coefficients of Propeller-hull Interaction in Propulsion System of Inland Waterway Vessels with Stern Tunnels. TransNav : International Journal on Marine Navigation and Safety of Sea Transportation, 8(3), 377-384. https://doi.org/10.12716/1001.08.03.08

[18] Khanfir, S., Hasegawa, K., Nagarajan, V., Shouji, K., Lee, S.K, 2011. Manoeuvring charateristics of twin-rudder sytems: rudder-hull interaction effect on the manoeuvrability of twin-rudder ships. Journal of Marine Science and Technology, 16(4), 472-490. https://doi.org/10.1007/s00773-011-0140-3

[19] Khanfir, S., Hasegawa, K., Kobayashi, E., Nagarajan, V., 2012. Mathematical Model for Manoeuvring of Twin-propeller Twin-rudder Ship Considering Peculiar Rudder Normal Force Phenomenon, Proc. MARSIM 2012, Singapore, Apr. 23-27.

[20] Inoue, S., Hirano, M., Kijima, K., Takashina, J.,1981. A practical calculation method of ship maneuvering motion. International Shipbuilding Progress, 28(325), 207-222. https://doi.org/10.3233/ISP$\underline{1981-2832502}$

[21] Lee S.K., Fujino, M., 2003. Assessment of mathematical model for the manoeuvring motion of a twinpropeller twin-rudder ship. International Shipbuilding Progress, 50,109-123.

[22] Yoshimura, Y., Ma, N., 2003. Manoeuvring prediction of fishing vessels. In: International Conference on Marine Simulation and Ship Maneuverability (MARSIM'03). Knazawa, Japan. Aug. http://hdl.handle.net/2115/757

[23] Hess, J. L., Smith, A., 1964. Calculation of Non-Lifting Potential Flow About Arbitrary ThreeDimensional Bodies. Journal of Ship Research, 8(4), 22-44.

[24] Newman J.N. Marine Hydrodynamics[M]. MIT Press, 1997:141-144.

[25] ITTC Recommended Procedures and Guidelines, 2014. Practical Guidelines for ship CFD Applications, NO. 7.5-03-02-03. https://ittc.info/media/4196/75-03-02-03.pdf

[26] Celik, I. B., Ghia, U., Roache, P. J., Freitas, C. J., Coleman, H., Raad, P. E., 2008. Procedure for estimation and reporting of uncertainty due to discretization in CFD applications. Journal of Fluids Engineering, 130(7): 078001. https://doi.org/10.1115/1.2960953

\footnotetext{
Submitted: 29.03.2021. Kun Dai, dkhue@126.com

College of Shipbuilding Engineering, Harbin Engineering University, Harbin

Accepted: $\quad$ 17.07.2021. 150001, China

Yunbo Li* (the corresponding author), multihull@163.com

College of Ocean Science and Engineering, Shanghai Maritime University, Shanghai 201306, China
} 